\title{
Prevalencia y factores socioeconómicos asociados al consumo de alcohol en jóvenes de hogares pobres en la subregión Montes de María, en el Caribe colombiano
}

\author{
Prevalence and Socioeconomic Factors Associated to Alcohol \\ Consumption by Youngsters from Low-income Homes in the Montes \\ de María Sub-region, in the Colombian Caribbean \\ Prevalência e fatores socioeconômicos associados ao consumo de \\ álcool em jovens de lares pobres na sub-região Montes María, no \\ Caribe colombiano
}

Fernando Enrique Salcedo-Mejia', Jossie Stefany Alvis-Zakzuk², Justo Jesús Paz-Wilches', Julian Patrick Manrique-Mclean', Marly Jerez-Arias ${ }^{5}$, Nelson Rafael Alvis-Guzmán ${ }^{6}$

1 Economista. Fundación Alzak, Cartagena, Colombia. fernandosalcedom@gmail.com

2 Economista. Fundación Alzak, Cartagena, Colombia. jossiealvis@hotmail.com

3 Médico y cirujano; epidemiólogo. Mutual Ser EPS, Colombia. jpaz@mutualser.org

4 Enfermero, magíster en Administración (MBA), especialista en Gestión Gerencial. Mutual Ser EPS, Colombia. jmanrique@mutualser.org

5 Odontóloga, especialista en Gerencia en Salud y Gerencia Financiera en servicios de salud. Fundación Sersocial, Colombia.mjerez@sersocial.org

6 Médico y cirujano, magíster en Salud Pública, doctor en Economía y Gestión de la Salud. Grupo de Investigación en Economía de la Salud (GIEs), Cartagena, Colombia, Universidad de Cartagena. Centro de Investigación y Docencia (CID). Hospital Infantil Napoleón Franco Pareja. nalvis@yahoo.com

Recibido: 13/07/2016. Aprobado: 18/05/2018. Publicado: 30/04/2018

Salcedo-Mejía FE, Alvis-Zakzuk JS, Paz-Wilches JJ, Manrique-Mclean JP, Jerez-Arias M, Alvis-Guzmán NR. Prevalencia y factores socioeconómicos asociados al consumo de alcohol en jóvenes de hogares pobres en la subregión Montes de María, en el Caribe colombiano. Rev. Fac. Nac. Salud Pública. 2018;36(2):37-48. Dor:10.17533/udea.rfnsp.v36n2a06 


\section{Resumen}

Objetivo: Estimar la prevalencia de consumo semanal de alcohol en población joven y sus determinantes socioeconómicos en la subregión Montes de María, del Caribe colombiano. Metodologías: Estudio analítico transversal de 10667 jóvenes encuestados de 10 a 24 años. Se utilizaron tablas de frecuencias y medidas de tendencia central, dispersión y posición, con estratificación de la muestra por sexo y grupos edad. Se usó un modelo logístico de respuesta dicotómica para la prevalecía, explorando factores de riesgo. Resultados: La prevalencia de consumo de alcohol de alguna vez por semana es 9,4\%. La prevalencia en hombres es $14,5 \%$ y en mujeres es $5,6 \%$. En menores de edad es $3,9 \%$ y en mayores de edad es 19,0\%. El municipio de San Onofre presenta la mayor prevalecía (14,9\%). Además, ser hombre, ser empleado, tener una mala relación con los padres, tener familiares víctimas del conflicto o asesorías psicológicas previas incrementó el riesgo de consumo de alcohol. En las mujeres, pero no en los hombres, tener pensamientos suicidas se relacionó con un mayor riesgo de con- sumo de alcohol. Para ambos sexos, tener de 18 a 24 años, perder años escolares, consumir sustancias psicoactivas y comportarse como agresor en casos de matoneo escolar incrementan el riesgo de consumo de alcohol. Discusión: La prevalencia de consumo de alcohol de alguna vez a la semana de los jóvenes de Montes de María está a 1,5 puntos porcentuales de la prevalencia de consumo perjudicial a nivel nacional (11,07\%). Los jóvenes de 18 a 24 años tienen una prevalencia igual al consumo de alcohol riesgoso y perjudicial nacional para el mismo rango de edad $(18,76 \%)$. Los factores de riesgo encontrados denotan problemas familiares, desinterés escolar y problemas psicológicos. Conclusiones: Hay alta prevalencia de consumo de alcohol en jóvenes de Montes de María, donde es necesario afianzar programas preventivos de consumo de alcohol en edades tempranas.

-Palabras clave: abuso de alcohol, consumo de bebidas alcohólicas, consumo de alcohol en jóvenes, factores de riesgo, trastornos inducidos por alcohol, conflicto armado.

\section{Abstract}

Objective: to estimate the prevalence of weekly alcohol composition in the young population and its socioeconomic determinants, in the Montes de María sub-region, Colombian Caribbean. Methodology: analytic cross-sectional study with 10667 surveys on youngsters aged 10-24 years. Frequency grids and measures of central tendency, dispersion and position were used, classifying the sample by sex and age groups. A logistic model of dichotomous questions was used for the prevalence, studying risk factors. Results: the prevalence of alcohol consumption at least once a week is $9.4 \%$. In males, the prevalence is $14.5 \%$, and in women, it is $5.6 \%$. Underage prevalence is $3.9 \%$ and adult prevalence is $19.0 \%$. The municipality of San Onofre has the highest prevalence (14.9\%). Additionally, the risk of alcohol consumption increased in male who were employees, had dysfunctional family relationship, relatives who were victims of the armed conflict or who received previous psychological attention. In women, but not in men, having suicidal thoughts was associated with higher risk of alcohol consumption. For all the participants, being between 18 and 24 years of age, having failed school years, consuming psychoactive substances being a school bully increased the risk of alcohol consumption. Discussion: the prevalence of atleast-once-a-week alcohol consumption in the youngsters of Montes de María is $1.5 \%$ below the national harmful alcohol consumption level (11.07\%). The sample's youngsters aged 18-24 years have the same prevalence of high-risk and harmful alcohol consumption as the national level for the same age range (18.76\%). The risk factors found reveal family issues, a lack of interest in school and psychological problems. Conclusions: there is a high prevalence of alcohol consumption in the youngsters of Montes de María and it is necessary to enforce programs to prevent alcohol consumption at young ages.

Key words: alcohol abuse, alcoholic beverage consumption, alcohol consumption by youngsters, risk factors, disorders originated by alcohol, armed conflict

\section{Resumo}

Objetivo: Estimar a prevalência de consumo semanal de álcool na população jovem e seus determinantes socioeconômicos na sub-região Montes de María, do Caribe colombiano. Metodologia: foi um estudo analítico transversal de 10667 jovens de 10 a 24 anos que foram indagados. Utilizaram-se grelhas de frequência e medidas de tendência central, dispersão e posição, com estratificação da amostra por sexo e grupos de idade. Utilizou-se um modelo logístico de resposta dicotómica para a prevalência, estudando fatores de risco. Resultados: a prevalência de con- sumo de álcool de alguma vez por semana é de 9,4\%. A prevalência em homes é $14,5 \%$ e 5,6\% em mulheres. Em menores de idade é 3,9\% e em adultos é 19,0\%. O município San Onofre tem a prevalência mais alta (14,9\%). Além disso, ser homem, ser empregado, ter uma relação ruim com os pais, ter família vítima do conflito ou assessorias psicológicas anteriores aumentou o risco de consumo de álcool. Nas mulheres, não nos homens, ter pensamentos suicidas esteve relacionado com mais risco de consumo de álcool. Para todos, ter entre 18 e 24 anis, perder anos da 
escola, consumir substâncias psicoativas e ter comportamento agressor em casos de bullying na escola aumentam o risco de consumo de álcool. Discussão: a prevalência do consumo de álcool de alguma vez por semana dos jovens de Montes de María fica a 1,5 pontos de percentagem da prevalência do consumo prejudicial a nível nacional (11,07\%). Os jovens de 18 a 24 anos têm uma prevalência igual ao consumo de álcool de risco e prejudicial nacional no mesmo intervalo de idade $(18,76 \%)$. Os fa- tores de risco achados revelam problemas familiares, desinteresse escolar e problemas psicológicos. Conclusões: há uma prevalência alta de consumo de álcool nos jovens de Montes de María, sendo necessário fortalecer programas de prevenção de consumo de álcool nas idades precoces.

-Palavras-chave: abuso de álcool, consumo de bebidas alcoólicas, consumo de álcool em jovens, fatores de risco, transtornos provocados pelo álcool, conflito armado

\section{Introducción}

El abuso de alcohol es un problema de salud pública, por las pérdidas sociales y económicas que genera tanto para las personas como para la sociedad en su conjunto. En 2012, unos 3,3 millones de defunciones (5,9 \% del total mundial) fueron atribuibles al consumo de alcohol, siéndole imputable el 5,1\% de la carga mundial de morbilidad y lesiones, además de representar el $25 \%$ de las defunciones y las discapacidades en edades de 20 a 39 años [1].

En población joven (15 a 24 años de edad), el abuso del alcohol se asocia con el acceso al consumo de otras sustancias psicoactivas, con tener relaciones sexuales no planificadas, con el riesgo de contraer el virus de la inmunodeficiencia humana (VIH), con provocar embarazos no deseados y con comportamientos agresivos o delictivos [2]. Se estima que Europa (69,5\%) y la región de las Américas (52,7 \%) tienen mayor proporción de bebedores adolescentes (15 a 19 años) que en otras regiones del mundo. Además, la prevalencia de consumo abusivo de alcohol entre los adolescentes es $11,7 \%$ a nivel mundial, siendo mayor que la prevalencia de bebedores entre 15 años y más $(7,5 \%)$. En la región de las Américas, el patrón de consumo abusivo del alcohol tiene una prevalecía del $18,4 \%$ para edades de 15 a 19 años, siendo mayor en hombres (29,3\%) [1].

El "Estudio nacional de consumo de sustancias psicoactivas en Colombia 2013" muestra que, en el país, el $87 \%$ de los encuestados ha consumido alcohol en algún momento de su vida, y el 35,8 \% manifiesta haber consumido en los últimos 30 días, siendo más frecuente en hombres (46,2 \%), y entre 18 y 24 años, con el 49,2 \%. El $31 \%$ de los consumidores de alcohol mensuales presenta patrones de consumo abusivo del mismo; por lo tanto, tiene mayor impacto entre la población joven [3].

La investigación de patrones de consumo de alcohol no se ha extendido a las subregiones del país, más aún a esas donde han existido cambios abruptos del tejido social, como los que genera la violencia armada. El trabajo de Hewitt et al. [4] pone en manifiesto dichos cambios en población escolar en zona rural del país, expuesta al conflicto armado. Se encontró que el $72 \%$ de la población presentó afectaciones psicológicas: el 64,4 \%, conductas internalizadas; el $47 \%$, conductas externalizadas en rango clínico; el $32 \%$, problemas somáticos; el $56 \%$ se hallaba en riesgo de estrés postraumático, y el $93 \%$ consumía alcohol en grado moderado. El estudio evidenció una alta necesidad de atención en salud.

A su vez, la investigación de Gantiva et al. [5] estima la prevalencia de consumo de sustancias psicoactivas en población de posconflicto, encontrando que el alcohol es la sustancia con mayor porcentaje de prevalencia a lo largo de la vida y durante el último mes. Se observó que el mayor porcentaje de personas inicia el consumo entre los 8 y 13 años, y que el $42 \%$ de los jóvenes ha tenido, por lo menos, un episodio de embriaguez.

El presente estudio explora la prevalencia de consumo de alcohol en población joven y sus determinantes socioeconómicos, en siete municipios de los Montes de María, de la región del Caribe colombiano.

\section{Metodología}

Se trata de un estudio observacional, analítico y trasversal, donde se estima la prevalencia y los factores de riesgo del consumo de alcohol de jóvenes de 10 a 24 años de ambos sexos afiliados a una entidad promotora de salud (EPS) del régimen subsidiado. Estos jóvenes pertenecen a siete municipios de la subregión de los Montes de María: Calamar, El Carmen de Bolívar, El Guamo, San Jacinto, San Juan Nepomuceno, San Onofre y Zambrano.

Los datos son derivados de la aplicación de un cuestionario elaborado e implementado por la fundación Sersocial, donde se recolectan datos de las características demográficas y psicosociales de la población de 10667 jóvenes de la subregión de los Montes de María en 2014. La encuesta fue aplicada de forma aleatoria, basados en la cantidad de afiliados en los municipios respectivos, buscando que fuera estadísticamente representativa de las poblaciones. La muestra representó el 30,2 y el 15,9\% de la población de 10 a 24 años asegurada y poblacional de 2014, respectivamente, para esos municipios.

El instrumento de captura muestra, en un primer segmento, las características demográficas y del entorno familiar del joven. El segundo segmento determina la percepción de los jóvenes encuestados hacia el consumo de sustancias adictivas, sustancias psicoactivas, 
sexualidad, violencia y conflicto armado, y matoneo escolar. El instrumento se sometió solo a validez facial con un grupo de pares investigadores en el área Materno Infantil y Salud Comunitaria. Con el objetivo de simplificar el análisis, las respuestas de escalas de percepción se convirtieron a variables dicotómicas en los que manifestaron algún grado de acuerdo.

De la encuesta se toma el "consumo de alcohol de alguna a una vez a la semana" como variable de interés. Se considera que existe el consumo de alcohol si tiene una percepción afirmativa. La estimación de la prevalencia puntual de consumo de alcohol una vez a la semanal es el cociente entre los casos afirmativos respecto la población encuestada. Esta prevalencia es discriminada por municipio, grupos de edad y sexo. Los grupos de edad fueron de 10 a 17 años y de 18 a 24, para poder observar las prevalencias de consumo en los menores de edad. Se reportan frecuencias absolutas, relativas e intervalos de confianza. La significancia de las diferencias entre grupos se realiza a través de un test ji-cuadrado $\left(X^{2}\right)$.

Se exploran las posibles asociaciones estadísticas para determinar factores de riesgo o protectores en el consumo de alcohol semanal. Se plantea un modelo logístico, donde la variable dependiente es el "consumo de alcohol alguna vez a la semana".

Las variables explicativas se dividen en características sociodemográficas del joven, características del hogar, factores psicológicos y del entorno.

En las características del joven se toman la edad, el sexo, nivel educativo, situación laboral y si ha tenido hijos.

Los factores psicológicos del joven se capturan, por un lado, de la situación de convivencia escolar, evidenciada en conductas agresivas; es aproximada a través del matoneo escolar, evaluando si el joven se comporta como agresor o agredido. Para ello, se hace explícita la situación "he amenazado y agredido física o verbalmente a mis compañeros de colegio" y "he sido víctima de agresiones físicas o verbales por parte de mis compañeros". Esto solo sería válido para los jóvenes en edad escolar. Se incluye si el joven ha pensado en suicidio tras haber asistido a asesorías psicológicas como aproximación a problemas depresivos, emocionales o de autoestima.

Entre las características del hogar se tienen en cuenta el ingreso económico del hogar, la educación de los padres y el autorreporte de la relación de los padres con el joven.

Por último, las variables como el departamento y el área de vivienda conforman el ámbito de entorno.

La modelación se realiza en un primer modelo conjunto y posteriormente se ajusta por sexo. Se reportan las pruebas de bondad de ajuste de Hosmer-Lemeshow como prueba de correcta especificación del modelo final, siendo estadísticamente significativo si $p \geq 0,05$. Se presentan los parámetros estimados del modelo en
Odds Ratio (OR). El procesamiento de la información y los análisis estadísticos se llevan a cabo en el software estadístico STATA ${ }^{\circledR}$ versión 13.

La aplicación del instrumento fue individual y se siguieron principios éticos de confidencialidad y anonimato, así como la firma del asentimiento y el consentimiento informado por el responsable legal del menor.

\section{Resultados}

En la tabla 1 se observan las características de los jóvenes encuestados. E1 56,2 \% de ellos fueron mujeres, con una media de edad general de 16,21 $( \pm 4,08)$ años. El $63,2 \%$ están en las edades de 10-17 años.

El nivel educativo predominante es la secundaria, con $71,08 \%$, con alrededor de $13 \%$ con pérdidas de años escolares. La educación de los padres reportada es principalmente de nivel de primaria.

Los encuestados viven principalmente en los municipios de El Carmen de Bolívar (34,95 \%), San Juan Nepomuceno (17,7\%), San Jacinto (12,9\%) y San Onofre $(12,06 \%)$. La situación laboral reportada es, en gran medida, desempleado, con ingresos familiares menores a un salario mínimo legal vigente (SMLV).

Un poco más de la mitad de los jóvenes encuestados se reconocen víctimas del conflicto armado, donde, a su vez, alrededor de un tercio de sus familiares se reconocen como víctimas.

Se encuentra que un poco menos de un tercio ha recibido asistencia psicológica y en la misma proporción han tenido pensamientos suicidas.

En la tabla 2 se reportan las prevalencias de "consumo de alcohol de alguna vez en la semana", discriminada por diferentes características del joven, familiares y geográficas.

Se calcula una prevalencia de consumo de alcohol $9,47 \%$ (IC $95 \%=8,91 \%-10 \%$ ) de "alguna vez a la semana", existiendo una diferencia entre la prevalencia de hombres y mujeres de 8,8 puntos porcentuales. Esta diferencia persiste en cada característica observada, variando en mayor o menor medida.

Se encuentra concentración del consumo de alcohol en los jóvenes de San Onofre (14,91 \%), con mayoría de edad $(19,01 \%)$, pérdida de 3 o más años escolares $(38,95 \%)$, comportamiento de agresor en caso de matoneo escolar $(18,1 \%)$, manifestación de consumo de algún tipo de sustancia psicoactiva $(37,75 \%)$ y pensamientos suicidas $(20,75 \%)$.

En la tabla 3 se muestran los factores de riesgo identificados en términos de oR con IC $95 \%$ y sus pruebas de ajustes.

Al controlar las variables observables en estudio, se estimó que el riesgo de consumir alcohol en la semana es tres veces mayor en hombres que en mujeres. Tanto 
Tabla 1. Características demográficas y socioeconómicas de la población encuestada.

\begin{tabular}{|c|c|c|c|c|c|}
\hline Variable & & Total & Masculino & Femenino & Test \\
\hline Número de encuestados & & 10677 & 4671 & 6006 & \\
\hline Edad (años) & & $16,21( \pm 4,08)$ & $15,93( \pm 4,03)$ & $16,42( \pm 4,1)$ & $p=0,000$ \\
\hline \multirow{4}{*}{ Escolaridad del joven } & Sin educación & 0,72 & 1,03 & 0,48 & \multirow{4}{*}{$p=0,000$} \\
\hline & Primaria & 22,21 & 26,5 & 18,86 & \\
\hline & Secundaria & 71,08 & 68,29 & 73,24 & \\
\hline & Superior & 5,99 & 4,17 & 7,41 & \\
\hline \multirow{3}{*}{ Situación laboral } & Desempleado & 46,12 & 42,32 & 49,07 & \multirow{3}{*}{$p=0,000$} \\
\hline & Empleado & 15,47 & 19,14 & 12,62 & \\
\hline & Inactivos & 38,41 & 38,54 & 38,31 & \\
\hline \multirow{2}{*}{ Víctima de conflicto } & No & 48,82 & 50,07 & 47,85 & \multirow{2}{*}{$p=0,023$} \\
\hline & Sí & 51,18 & 49,93 & 52,15 & \\
\hline \multirow{2}{*}{$\begin{array}{l}\text { Uso de sustancias } \\
\text { psicoactivas }\end{array}$} & No & 98,09 & 97,2 & 98,78 & \multirow{2}{*}{$p=0,000$} \\
\hline & Sí & 1,91 & 2,8 & 1,22 & \\
\hline \multirow{4}{*}{ Pérdida de años escolares } & Nunca & 86,83 & 82,53 & 90,18 & \multirow{4}{*}{$p=0,000$} \\
\hline & 1 año & 10,06 & 12,46 & 8,19 & \\
\hline & 2 años & 2,22 & 3,43 & 1,28 & \\
\hline & 3 o más años & 0,89 & 1,58 & 0,35 & \\
\hline \multirow{4}{*}{ Escolaridad padre } & Sin educación & 21,01 & 20,66 & 21,28 & \multirow{4}{*}{$p=0,008$} \\
\hline & Primaria & 47,13 & 45,73 & 48,22 & \\
\hline & Secundaria & 29,02 & 30,59 & 27,79 & \\
\hline & Superior & 2,85 & 3,02 & 2,71 & \\
\hline \multirow{4}{*}{ Escolaridad de la madre } & Sin educación & 15,26 & 15,05 & 15,42 & \multirow{4}{*}{$p=0,015$} \\
\hline & Primaria & 47,11 & 45,86 & 48,09 & \\
\hline & Secundaria & 34,29 & 35,3 & 33,5 & \\
\hline & Superior & 3,34 & 3,79 & 3 & \\
\hline \multirow{3}{*}{ Ingresos familiares } & $<1 \mathrm{SMLV}$ & 94,53 & 94,56 & 94,51 & \multirow{3}{*}{$p=0,984$} \\
\hline & $1 \mathrm{SMLV}$ & 4,9 & 4,86 & 4,93 & \\
\hline & $>1$ SMLV & 0,57 & 0,58 & 0,57 & \\
\hline \multirow{2}{*}{ Relación padres-joven } & Mala & 9,03 & 9 & 9,06 & \multirow{2}{*}{$p=0,905$} \\
\hline & Buena & 90,97 & 91 & 90,94 & \\
\hline \multirow{2}{*}{ Relación padre-joven } & Mala & 10,92 & 10,72 & 11,08 & \multirow{2}{*}{$p=0,572$} \\
\hline & Buena & 89,08 & 89,28 & 88,92 & \\
\hline \multirow{2}{*}{ Relación madre-joven } & Mala & 5,9 & 5,81 & 5,97 & \multirow{2}{*}{$p=0,725$} \\
\hline & Buena & 94,1 & 94,19 & 94,03 & \\
\hline \multirow{2}{*}{ Maltrato físico } & No & 98,87 & 99,03 & 98,74 & \multirow{2}{*}{$p=0,175$} \\
\hline & Sí & 1,13 & 0,97 & 1,26 & \\
\hline \multirow{2}{*}{ Maltrato verbal } & No & 97,71 & 97,93 & 97,54 & $n$ \\
\hline & Sí & 2,29 & 2,07 & 2,46 & $\mu-0,200$ \\
\hline Familiar víctima del conflicto & No & 66,99 & 66,11 & 67,67 & 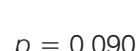 \\
\hline & Sí & 33,01 & 33,89 & 32,33 & $r=0,000$ \\
\hline Asesoría nsicoló & No & 69,99 & 70,35 & 69,71 & \\
\hline Asesonla psicologica & Sí & 30,01 & 29,65 & 30,29 & J,4t \\
\hline
\end{tabular}


Continuación Tabla 1

\begin{tabular}{|c|c|c|c|c|c|}
\hline Variable & & Total & Masculino & Femenino & Test \\
\hline \multirow{2}{*}{$\begin{array}{l}\text { Agresor en casos de manoteo } \\
\text { escolar }\end{array}$} & No & 94,77 & 93,43 & 95,82 & \multirow{2}{*}{$p=0,000$} \\
\hline & Sí & 5,23 & 6,57 & 4,18 & \\
\hline \multirow{2}{*}{ Pensamientos suicidas } & No & 98,01 & 98,67 & 97,5 & \multirow{2}{*}{$p=0,000$} \\
\hline & Sí & 1,99 & 1,33 & 2,5 & \\
\hline \multirow{2}{*}{$\begin{array}{l}\text { Amigos influencian mis } \\
\text { decisiones }\end{array}$} & No & 67,57 & 66,54 & 68,36 & \multirow{2}{*}{$p=0,045$} \\
\hline & Sí & 32,43 & 33,46 & 31,64 & \\
\hline \multirow{7}{*}{ Municipios } & Calamar & 7,72 & 6,98 & 8,29 & \multirow{7}{*}{$p=0,000$} \\
\hline & $\begin{array}{l}\text { El Carmen de } \\
\text { Bolívar }\end{array}$ & 34,95 & 33,85 & 35,81 & \\
\hline & El Guamo & 8,82 & 10,25 & 7,71 & \\
\hline & San Jacinto & 12,94 & 13,53 & 12,49 & \\
\hline & $\begin{array}{l}\text { San Juan } \\
\text { Nepomuceno }\end{array}$ & 17,7 & 18,11 & 17,38 & \\
\hline & San Onofre & 12,06 & 10,98 & 12,9 & \\
\hline & Zambrano & 5,8 & 6,29 & 5,41 & \\
\hline \multirow{2}{*}{ Área vivienda } & Rural & 15,26 & 15,89 & 14,77 & \multirow{2}{*}{$p=0,111$} \\
\hline & Urbana & 84,74 & 84,11 & 85,23 & \\
\hline
\end{tabular}

SMLV: Salario mínimo legal vigente; $p$ : valor de la probabilidad.

Tabla 2. Prevalencias (\%) e intervalos de confianza al $95 \%$ del "consumo de alcohol alguna vez a la semana" en función de variables sociodemográficas.

\begin{tabular}{|c|c|c|c|c|c|c|c|c|}
\hline \multirow{2}{*}{\multicolumn{2}{|c|}{ Variable }} & \multirow{2}{*}{ Número } & \multicolumn{2}{|c|}{ Masculino } & \multicolumn{2}{|c|}{ Femenino } & \multicolumn{2}{|c|}{ Total } \\
\hline & & & $\%$ & IC $95 \%$ & $\%$ & IC $95 \%$ & $\%$ & IC $95 \%$ \\
\hline Total & & 10677 & 14,47 & {$[13,46-15,48]$} & 5,58 & {$[5,00-6,16]$} & 9,47 & {$[8,91-10,02]$} \\
\hline \multirow{2}{*}{$\begin{array}{l}\text { Rangos de } \\
\text { edades }\end{array}$} & $10-17$ & 6748 & 5,78 & {$[4,95-6,60]$} & 2,34 & {$[1,85-2,83]$} & 3,91 & {$[3,45-4,37]$} \\
\hline & $18-24$ & 3929 & 31,3 & {$[29,02-33,58]$} & 10,65 & {$[9,40-11,90]$} & 19,01 & {$[17,79-20,24]$} \\
\hline \multirow{4}{*}{$\begin{array}{l}\text { Escolaridad del } \\
\text { joven }\end{array}$} & Sin educación & 77 & 14,58 & {$[4,60-24,57]$} & 0 & {$[0,00-0,00]$} & 9,09 & {$[2,67-15,51]$} \\
\hline & Primaria & 2371 & 7,84 & {$[6,34-9,33]$} & 2,91 & {$[1,93-3,89]$} & 5,48 & {$[4,57-6,40]$} \\
\hline & Secundaria & 7589 & 16,39 & {$[15,11-17,68]$} & 6 & {$[5,30-6,70]$} & 10,37 & {$[9,68-11,06]$} \\
\hline & Superior & 640 & 25,13 & {$[19,04-31,22]$} & 8,54 & {$[5,94-11,14]$} & 13,59 & {$[10,94-16,25]$} \\
\hline \multirow{3}{*}{$\begin{array}{l}\text { Situación } \\
\text { laboral }\end{array}$} & Desempleado & 4924 & 15,23 & {$[13,64-16,81]$} & 6,07 & {$[5,21-6,94]$} & 9,75 & {$[8,92-10,58]$} \\
\hline & Empleado & 1652 & 26,51 & {$[23,62-29,40]$} & 7,52 & {$[5,64-9,40]$} & 17,8 & {$[15,95-19,64]$} \\
\hline & Inactivos & 4101 & 7,67 & {$[6,44-8,90]$} & 4,3 & {$[3,47-5,13]$} & 5,78 & {$[5,06-6,49]$} \\
\hline \multirow{2}{*}{$\begin{array}{l}\text { Víctima de } \\
\text { conflicto }\end{array}$} & No & 5213 & 14,19 & {$[12,78-15,61]$} & 5,36 & {$[4,53-6,18]$} & 9,32 & {$[8,53-10,11]$} \\
\hline & Sí & 5464 & 14,75 & {$[13,31-16,19]$} & 5,78 & {$[4,96-6,60]$} & 9,61 & {$[8,83-10,39]$} \\
\hline \multirow{2}{*}{$\begin{array}{l}\text { Uso de } \\
\text { sustancias }\end{array}$} & No & 10473 & 13,59 & {$[12,59-14,59]$} & 5,34 & {$[4,77-5,92]$} & 8,92 & {$[8,37-9,46]$} \\
\hline & Sí & 204 & 45,04 & {$[36,52-53,56]$} & 24,66 & {$[14,77-34,55]$} & 37,75 & {$[31,09-44,40]$} \\
\hline \multirow{4}{*}{$\begin{array}{l}\text { Pérdida de } \\
\text { años escolares }\end{array}$} & Nunca & 9271 & 12,11 & {$[11,08-13,14]$} & 5,21 & {$[4,62-5,80]$} & 8,08 & {$[7,52-8,63]$} \\
\hline & 1 año & 1074 & 21,65 & {$[18,30-25,00]$} & 7,93 & {$[5,54-10,31]$} & 15,36 & {$[13,21-17,52]$} \\
\hline & 2 años & 237 & 30 & {$[22,90-37,10]$} & 15,58 & {$[7,48-23,69]$} & 25,32 & {$[19,78-30,85]$} \\
\hline & 3 o más años & 95 & 47,3 & {$[35,92-58,67]$} & 9,52 & {$[-3,03-22,08]$} & 38,95 & {$[29,14-48,75]$} \\
\hline \multirow{4}{*}{$\begin{array}{l}\text { Escolaridad } \\
\text { padre }\end{array}$} & Sin educación & 2243 & 17,2 & {$[14,82-19,58]$} & 6,81 & {$[5,43-8,19]$} & 11,28 & {$[9,97-12,59]$} \\
\hline & Primaria & 5032 & 15,03 & {$[13,51-16,54]$} & 6,22 & {$[5,34-7,09]$} & 9,96 & {$[9,13-10,78]$} \\
\hline & Secundaria & 3098 & 12,04 & {$[10,35-13,72]$} & 3,48 & {$[2,60-4,35]$} & 7,42 & {$[6,50-8,35]$} \\
\hline & Superior & 304 & 12,06 & {$[6,68-17,43]$} & 6,13 & {$[2,45-9,82]$} & 8,88 & {$[5,68-12,08]$} \\
\hline
\end{tabular}


Continuación Tabla 2

\begin{tabular}{|c|c|c|c|c|c|c|c|c|}
\hline \multirow{2}{*}{\multicolumn{2}{|c|}{ Variable }} & \multirow{2}{*}{ Número } & \multicolumn{2}{|c|}{ Masculino } & \multicolumn{2}{|c|}{ Femenino } & \multicolumn{2}{|c|}{ Total } \\
\hline & & & $\%$ & IC $95 \%$ & $\%$ & IC $95 \%$ & $\%$ & IC $95 \%$ \\
\hline \multirow{4}{*}{$\begin{array}{l}\text { Escolaridad de } \\
\text { la madre }\end{array}$} & Sin educación & 1629 & 17,35 & {$[14,55-20,15]$} & 5,83 & {$[4,32-7,34]$} & 10,8 & {$[9,30-12,31]$} \\
\hline & Primaria & 5030 & 15,27 & {$[13,74-16,79]$} & 6,37 & {$[5,48-7,26]$} & 10,16 & {$[9,32-10,99]$} \\
\hline & Secundaria & 3661 & 12,61 & {$[11,01-14,22]$} & 4,37 & {$[3,48-5,27]$} & 8,09 & {$[7,20-8,97]$} \\
\hline & Superior & 357 & 10,73 & {$[6,17-15,30]$} & 5 & {$[1,82-8,18]$} & 7,84 & {$[5,05-10,63]$} \\
\hline \multirow{3}{*}{$\begin{array}{l}\text { Ingresos } \\
\text { familiares }\end{array}$} & $<1$ SMLV & 10093 & 14,35 & {$[13,32-15,39]$} & 5,44 & {$[4,85-6,03]$} & 9,34 & {$[8,78-9,91]$} \\
\hline & $1 \mathrm{smLV}$ & 523 & 17,18 & {$[12,27-22,09]$} & 7,77 & {$[4,72-10,82]$} & 11,85 & {$[9,08-14,63]$} \\
\hline & $>1$ SMLV & 61 & 11,11 & {$[-0,74-22,97]$} & 8,82 & {$[-0,71-18,36]$} & 9,84 & {$[2,36-17,31]$} \\
\hline \multirow{2}{*}{$\begin{array}{l}\text { Relación } \\
\text { padres-joven }\end{array}$} & Mala & 961 & 19,09 & {$[15,33-22,86]$} & 10,52 & {$[7,93-13,10]$} & 14,26 & {$[12,05-16,47]$} \\
\hline & Buena & 9678 & 13,99 & {$[12,94-15,03]$} & 5,04 & {$[4,46-5,62]$} & 8,96 & {$[8,39-9,53]$} \\
\hline \multirow{2}{*}{$\begin{array}{l}\text { Relación padre- } \\
\text { joven }\end{array}$} & Mala & 1115 & 17,99 & {$[14,55-21,44]$} & 9,89 & {$[7,57-12,21]$} & 13,36 & {$[11,37-15,36]$} \\
\hline & Buena & 9093 & 14,1 & {$[13,02-15,18]$} & 4,99 & {$[4,39-5,58]$} & 8,97 & {$[8,39-9,56]$} \\
\hline \multirow{2}{*}{$\begin{array}{l}\text { Relación } \\
\text { madre-joven }\end{array}$} & Mala & 622 & 17,54 & {$[12,98-22,09]$} & 10,45 & {$[7,26-13,64]$} & 13,5 & {$[10,82-16,19]$} \\
\hline & Buena & 9918 & 14,22 & {$[13,18-15,26]$} & 5,15 & {$[4,57-5,73]$} & 9,12 & {$[8,56-9,69]$} \\
\hline \multirow{2}{*}{ Maltrato físico } & No & 9456 & 14,67 & {$[13,60-15,74]$} & 5,47 & {$[4,85-6,08]$} & 9,56 & {$[8,97-10,15]$} \\
\hline & Sí & 108 & 14,63 & {$[3,81-25,45]$} & 11,94 & {$[4,17-19,71]$} & 12,96 & {$[6,63-19,30]$} \\
\hline \multirow{2}{*}{ Maltrato verbal } & No & 9447 & 14,43 & {$[13,37-15,49]$} & 5,44 & {$[4,82-6,05]$} & 9,44 & {$[8,85-10,03]$} \\
\hline & Sí & 221 & 21,35 & {$[12,83-29,86]$} & 11,36 & {$[5,95-16,78]$} & 15,38 & {$[10,63-20,14]$} \\
\hline \multirow{2}{*}{$\begin{array}{l}\text { Familiar víctima } \\
\text { del conflicto }\end{array}$} & No & 7152 & 6,64 & {$[5,76-7,52]$} & 3,37 & {$[2,82-3,93]$} & 4,78 & {$[4,29-5,28]$} \\
\hline & Sí & 3525 & 29,75 & {$[27,50-32,01]$} & 10,2 & {$[8,85-11,54]$} & 18,98 & {$[17,68-20,27]$} \\
\hline \multirow{2}{*}{$\begin{array}{l}\text { Asesoría } \\
\text { psicológica }\end{array}$} & No & 7473 & 13,12 & {$[11,96-14,27]$} & 5,21 & {$[4,53-5,88]$} & 8,68 & {$[8,05-9,32]$} \\
\hline & Sí & 3204 & 17,69 & {$[15,68-19,70]$} & 6,43 & {$[5,30-7,56]$} & 11,3 & {$[10,20-12,39]$} \\
\hline \multirow{2}{*}{$\begin{array}{l}\text { Agresor en } \\
\text { casos de } \\
\text { manoteo } \\
\text { escolar }\end{array}$} & No & 10119 & 13,86 & {$[12,84-14,89]$} & 5,3 & {$[4,72-5,88]$} & 8,99 & {$[8,44-9,55]$} \\
\hline & Sí & 558 & 23,13 & {$[18,41-27,84]$} & 11,95 & {$[7,94-15,97]$} & 18,1 & {$[14,91-21,30]$} \\
\hline \multirow{2}{*}{$\begin{array}{l}\text { Pensamientos } \\
\text { suicidas }\end{array}$} & No & 10465 & 14,23 & {$[13,22-15,24]$} & 5,31 & {$[4,74-5,89]$} & 9,24 & {$[8,69-9,80]$} \\
\hline & Sí & 212 & 32,26 & {$[20,62-43,90]$} & 16 & {$[10,13-21,87]$} & 20,75 & {$[15,29-26,21]$} \\
\hline \multirow{2}{*}{$\begin{array}{l}\text { Amigos } \\
\text { influencian mis } \\
\text { decisiones }\end{array}$} & No & 7214 & 13,42 & {$[12,22-14,62]$} & 5,09 & {$[4,42-5,76]$} & 8,68 & {$[8,03-9,33]$} \\
\hline & Sí & 3463 & 16,57 & {$[14,73-18,41]$} & 6,63 & {$[5,51-7,75]$} & 11,12 & {$[10,07-12,16]$} \\
\hline \multirow{7}{*}{ Municipios } & Calamar & 824 & 14,11 & {$[10,33-17,89]$} & 2,41 & {$[1,06-3,76]$} & 7,04 & {$[5,29-8,79]$} \\
\hline & $\begin{array}{l}\text { El Carmen de } \\
\text { Bolívar }\end{array}$ & 3732 & 8,67 & {$[7,28-10,05]$} & 4,46 & {$[3,59-5,34]$} & 6,24 & {$[5,47-7,02]$} \\
\hline & El Guamo & 942 & 18,58 & {$[15,10-22,06]$} & 6,26 & {$[4,06-8,47]$} & 12,53 & {$[10,41-14,64]$} \\
\hline & San Jacinto & 1382 & 15,82 & {$[12,98-18,67]$} & 6,93 & {$[5,12-8,75]$} & 11 & {$[9,35-12,65]$} \\
\hline & $\begin{array}{l}\text { San Juan } \\
\text { Nepomuceno }\end{array}$ & 1890 & 15,01 & {$[12,60-17,42]$} & 5,94 & {$[4,50-7,37]$} & 10 & {$[8,65-11,35]$} \\
\hline & San Onofre & 1288 & 22,61 & {$[18,99-26,23]$} & 9,81 & {$[7,71-11,90]$} & 14,91 & {$[12,96-16,85]$} \\
\hline & Zambrano & 619 & 20,75 & {$[16,11-25,38]$} & 2,46 & {$[0,78-4,15]$} & 11,15 & {$[8,67-13,63]$} \\
\hline \multirow{2}{*}{ Área vivienda } & Rural & 1629 & 12,53 & {$[10,15-14,92]$} & 4,17 & {$[2,86-5,49]$} & 7,98 & {$[6,66-9,30]$} \\
\hline & Urbana & 9048 & 14,84 & {$[13,73-15,95]$} & 5,82 & {$[5,18-6,46]$} & 9,74 & {$[9,13-10,35]$} \\
\hline
\end{tabular}


Tabla 3. Modelo logístico para factores de riesgo del consumo de alcohol una vez por semana.

\begin{tabular}{|c|c|c|c|c|c|c|c|}
\hline \multirow{2}{*}{ Variable } & & \multicolumn{2}{|c|}{ Total } & \multicolumn{2}{|c|}{ Masculino } & \multicolumn{2}{|c|}{ Femenino } \\
\hline & & OR & IC $95 \%$ & OR & IC $95 \%$ & OR & IC $95 \%$ \\
\hline \multirow{2}{*}{ Sexo } & Femenino & 1 & & & & & \\
\hline & Masculino & $3,22^{\ddagger}$ & {$[2,72-3,81]$} & & & 1 & \\
\hline \multirow{2}{*}{ Rangos de edades } & $10-17$ & 1 & & 1 & & 1 & \\
\hline & $18-24$ & $4,08^{\ddagger}$ & {$[3,21-5,17]$} & $3,80^{\ddagger}$ & {$[2,85-5,07]$} & $4,93^{\ddagger}$ & {$[3,28-7,41]$} \\
\hline \multirow{4}{*}{ Escolaridad del joven } & Sin educación & 1 & & 1 & & 1 & \\
\hline & Primaria & 0,96 & {$[0,4-2,3]$} & 0,77 & {$[0,29-2,04]$} & 0,68 & {$[0,37-1,22]$} \\
\hline & Secundaria & 1,44 & {$[0,61-3,42]$} & 1,12 & {$[0,43-2,92]$} & 1,06 & {$[0,69-1,62]$} \\
\hline & Superior & 1,12 & {$[0,45-2,77]$} & 0,74 & {$[0,26-2,13]$} & 1 & \\
\hline \multirow{3}{*}{ Situación laboral } & Desempleado & 1 & & 1 & & 1 & \\
\hline & Empleado & $1,55^{\ddagger}$ & {$[1,28-1,88]$} & $1,76^{\ddagger}$ & {$[1,37-2,25]$} & 1,2 & {$[0,83-1,73]$} \\
\hline & Inactivos & 0,92 & {$[0,74-1,14]$} & 0,79 & {$[0,59-1,05]$} & 1,29 & {$[0,93-1,79]$} \\
\hline \multirow{2}{*}{ Víctima de conflicto } & No & 1 & & 1 & & 1 & \\
\hline & Sí & 0,96 & {$[0,81-1,13]$} & 0,99 & {$[0,8-1,23]$} & 0,9 & {$[0,68-1,18]$} \\
\hline \multirow{2}{*}{ Uso de sustancias } & No & 1 & & 1 & & 1 & \\
\hline & Sí & $2,88^{\ddagger}$ & {$[1,91-4,33]$} & $2,76^{\ddagger}$ & {$[1,68-4,54]$} & $3,33^{\ddagger}$ & {$[1,66-6,68]$} \\
\hline \multirow{4}{*}{ Pérdida de años escolares } & Nunca & 1 & & 1 & & 1 & \\
\hline & 1 año & 1,13 & {$[0,9-1,42]$} & 1,22 & {$[0,92-1,61]$} & 0,89 & {$[0,57-1,39]$} \\
\hline & 2 años & $2,03^{\ddagger}$ & {$[1,34-3,07]$} & $1,66^{\dagger}$ & {$[1,03-2,66]$} & $3,45^{\ddagger}$ & {$[1,68-7,07]$} \\
\hline & 3 o más años & $3,84^{\ddagger}$ & {$[2,12-6,95]$} & $4,64^{\ddagger}$ & {$[2,45-8,79]$} & 1,3 & {$[0,12-14,21]$} \\
\hline \multirow{4}{*}{ Escolaridad padre } & Sin educación & 1 & & 1 & & 1 & \\
\hline & Primaria & 0,94 & {$[0,75-1,17]$} & 1,05 & {$[0,79-1,4]$} & 0,74 & {$[0,52-1,06]$} \\
\hline & Secundaria & $0,72^{\dagger}$ & {$[0,55-0,95]$} & 0,81 & {$[0,57-1,13]$} & $0,55^{\dagger}$ & {$[0,34-0,88]$} \\
\hline & Superior & 0,93 & {$[0,54-1,61]$} & 0,77 & {$[0,38-1,55]$} & 1,26 & {$[0,53-3,01]$} \\
\hline \multirow{4}{*}{ Escolaridad de la madre } & Sin educación & 1 & & 1 & & 1 & \\
\hline & Primaria & 1,03 & {$[0,81-1,32]$} & 0,83 & {$[0,61-1,13]$} & $1,51^{*}$ & {$[0,98-2,33]$} \\
\hline & Secundaria & 1,13 & {$[0,86-1,49]$} & 1 & {$[0,71-1,42]$} & 1,4 & {$[0,86-2,29]$} \\
\hline & Superior & 0,75 & {$[0,41-1,36]$} & 0,74 & {$[0,35-1,54]$} & 0,67 & {$[0,21-2,19]$} \\
\hline \multirow{3}{*}{ Ingresos familiares } & $<1$ SMLV & 1 & & 1 & & 1 & \\
\hline & $1 \mathrm{SMLV}$ & 1,2 & {$[0,84-1,71]$} & 1,21 & {$[0,75-1,94]$} & 1,37 & {$[0,78-2,4]$} \\
\hline & $>1 \mathrm{SMLV}$ & 0,68 & {$[0,2-2,26]$} & 0,41 & {$[0,08-2,02]$} & 1,29 & {$[0,27-6,21]$} \\
\hline \multirow{2}{*}{ Relación padres-joven } & Buena & 1 & & 1 & & 1 & \\
\hline & Mala & $1,49^{\dagger}$ & {$[1,08-2,04]$} & $1,61^{\dagger}$ & {$[1,05-2,46]$} & 1,47 & {$[0,92-2,36]$} \\
\hline \multirow{2}{*}{ Relación padre-joven } & Mala & 1 & & 1 & & 1 & \\
\hline & Buena & 1,02 & {$[0,76-1,38]$} & 0,79 & {$[0,53-1,16]$} & 1,4 & {$[0,9-2,17]$} \\
\hline \multirow{2}{*}{ Relación madre-joven } & Mala & 1 & & 1 & & 1 & \\
\hline & Buena & 1,09 & {$[0,73-1,65]$} & 0,93 & {$[0,52-1,64]$} & 1,31 & {$[0,74-2,33]$} \\
\hline \multirow{2}{*}{ Maltrato físico } & No & 1 & & 1 & & 1 & \\
\hline & Sí & 1,04 & {$[0,47-2,29]$} & 0,8 & {$[0,26-2,45]$} & 1,1 & {$[0,27-4,55]$} \\
\hline \multirow{2}{*}{ Maltrato verbal } & No & 1 & & 1 & & 1 & \\
\hline & Sí & 1,25 & {$[0,71-2,2]$} & 1,38 & {$[0,68-2,79]$} & 1,13 & {$[0,37-3,45]$} \\
\hline \multirow{2}{*}{$\begin{array}{l}\text { Familiar víctima del } \\
\text { conflicto }\end{array}$} & No & 1 & & 1 & & 1 & \\
\hline & Sí & $1,83^{\ddagger}$ & {$[1,47-2,27]$} & $2,42^{\ddagger}$ & {$[1,84-3,19]$} & 1,15 & {$[0,81-1,64]$} \\
\hline
\end{tabular}


Continuación Tabla 3

\begin{tabular}{|c|c|c|c|c|c|c|c|}
\hline \multirow{2}{*}{\multicolumn{2}{|c|}{ Variable }} & \multicolumn{2}{|c|}{ Total } & \multicolumn{2}{|c|}{ Masculino } & \multicolumn{2}{|c|}{ Femenino } \\
\hline & & OR & IC $95 \%$ & OR & IC $95 \%$ & OR & IC $95 \%$ \\
\hline \multirow{2}{*}{ Asesoría psicológica } & No & 1 & & 1 & & 1 & \\
\hline & Sí & $1,27^{\dagger}$ & {$[1,07-1,51]$} & $1,37^{\dagger}$ & {$[1,09-1,73]$} & 1,15 & {$[0,87-1,51]$} \\
\hline \multirow{2}{*}{$\begin{array}{l}\text { Agresor en casos de } \\
\text { manoteo escolar }\end{array}$} & No & 1 & & 1 & & 1 & \\
\hline & Sí & $1,64^{\dagger}$ & {$[1,21-2,24]$} & $1,50^{\dagger}$ & {$[1,03-2,19]$} & $1,97^{\dagger}$ & {$[1,17-3,34]$} \\
\hline \multirow{2}{*}{ Pensamientos suicidas } & No & 1 & & 1 & & 1 & \\
\hline & Sí & $2,12^{\dagger}$ & {$[1,26-3,55]$} & 1,29 & {$[0,57-2,88]$} & $2,95^{\ddagger}$ & {$[1,65-5,26]$} \\
\hline \multirow{2}{*}{$\begin{array}{l}\text { Amigos influencian mis } \\
\text { decisiones }\end{array}$} & No & 1 & & 1 & & 1 & \\
\hline & Sí & 1,13 & {$[0,95-1,35]$} & $1,24^{*}$ & {$[0,98-1,57]$} & 0,98 & {$[0,73-1,32]$} \\
\hline \multirow{7}{*}{ Municipios } & Calamar & 1 & & 1 & & 1 & \\
\hline & $\begin{array}{l}\text { El Carmen de } \\
\text { Bolívar }\end{array}$ & $0,67^{*}$ & {$[0,44-1,02]$} & $0,40^{\ddagger}$ & {$[0,24-0,67]$} & 2,13 & {$[0,8-5,7]$} \\
\hline & El Guamo & $1,52^{*}$ & {$[0,98-2,38]$} & 1,23 & {$[0,72-2,1]$} & $3,08^{+}$ & {$[1,07-8,86]$} \\
\hline & San Jacinto & 1,15 & {$[0,74-1,79]$} & 0,88 & {$[0,51-1,51]$} & $2,88^{+}$ & {$[1,05-7,89]$} \\
\hline & $\begin{array}{l}\text { San Juan } \\
\text { Nepomuceno }\end{array}$ & 1,15 & {$[0,75-1,77]$} & 0,91 & {$[0,54-1,55]$} & $2,42^{*}$ & {$[0,88-6,61]$} \\
\hline & San Onofre & $2,40^{\ddagger}$ & {$[1,56-3,68]$} & $1,99^{\dagger}$ & {$[1,16-3,42]$} & $4,95^{\dagger}$ & {$[1,83-13,38]$} \\
\hline & Zambrano & 1,11 & {$[0,69-1,81]$} & 1,1 & {$[0,61-1,97]$} & 1,03 & {$[0,3-3,52]$} \\
\hline \multirow{2}{*}{ Área vivienda } & Rural & 1 & & 1 & & 1 & \\
\hline & Urbana & 1,22 & {$[0,96-1,55]$} & 1,12 & {$[0,84-1,51]$} & 1,42 & {$[0,92-2,19]$} \\
\hline \multicolumn{2}{|l|}{$\mathrm{R}^{2}$} & \multicolumn{2}{|c|}{0,21} & \multicolumn{2}{|c|}{0,23} & \multicolumn{2}{|c|}{0,14} \\
\hline \multicolumn{2}{|l|}{ N } & \multicolumn{2}{|c|}{9011} & \multicolumn{2}{|c|}{3997} & \multicolumn{2}{|c|}{4991} \\
\hline \multicolumn{2}{|c|}{ Hosmer-Lemeshow Prob $>X^{2}(98)$} & \multicolumn{2}{|r|}{0,87} & \multicolumn{2}{|c|}{0,28} & \multicolumn{2}{|c|}{0,14} \\
\hline
\end{tabular}

para el total como por sexo, el riesgo de consumo de alcohol en la semana es mayor en las edades de 18 a 24 años, observándose un riesgo mayor en mujeres.

Estar empleado implica una mayor probabilidad en el consumo solo presente en los hombres.

A su vez, para el total de muestra y ambos sexos, haber tenido mayor número de pérdida de años escolares incrementa el riesgo de consumo de alcohol.

En el caso de la educación de los padres, solo con respecto al padre y en la muestra total se observa una disminución del riesgo a medida que incrementa el nivel de educación.

Se observó que los jóvenes que manifiestan tener una mala relación con los padres tienen más riesgo de consumo de alcohol en la muestra total y en el grupo de hombres.

Además, se halló un incremento en el riesgo significativo del consumo de alcohol en los jóvenes con familiares víctimas de conflicto armado en el grupo agregado y los hombres.
Por otra parte, el consumo de alguna sustancia psicoactiva estuvo ligado a un mayor riesgo de consumo de alcohol en casi tres veces tanto en la muestra total como por sexo. De igual forma, presentar pensamientos suicidas incrementa el riesgo de consumo de alcohol a dos veces, y solo en el grupo de mujeres casi a tres veces de manera significativa. Las conductas agresivas en caso de matoneo escolar incrementarían en riesgo de consumo en el grupo total de muestra, así como en los hombres, observándose el mismo efecto significativo en el riesgo de consumo y del haber asistido a asesorías psicológicas.

Por último, se observa que el riego de "consumo de alcohol de alguna vez por semana" varía significativamente entre municipios, siendo el caso de San Onofre el que manifiesta un incremento en la probabilidad de consumo, tanto en el grupo total como por sexo. 


\section{Discusión}

Los principales hallazgos de este estudio fueron: la prevalencia de "consumo de alcohol de alguna vez a la semana" de los jóvenes de los Montes de María fue 9,47 \%: entre los varones, $14,47 \%$, y mujeres, $5,58 \%$. Por edad, la prevalencia de los jóvenes de 10 a 17 años fue de 3,91 \%, y de 18 a 24 , de 19,01\%. El grupo específico de hombres de 18 a 24 años presentó una prevalencia de "consumo de alcohol a la semana" de 31,3\%. El departamento de San Onofre tiene la mayor prevalencia general, con 14,91\%.

Además, ser hombre, ser empleado, tener una mala relación con los padres, tener familiares víctimas del conflicto, considerar que los amigos influyen en sus decisiones y haber tenido asesorías psicológicas fueron factores que se constituyeron en un mayor riesgo de consumo de alcohol. Lo fue también en las mujeres, pero no en los hombres, tener pensamientos suicidas.

Para ambos sexos, tener entre 18 y 24 años, perder años escolares, consumir sustancias psicoactivas y comportarse como agresor en casos de matoneo escolar incrementan el riesgo de consumo de alcohol.

De acuerdo con estos resultados, la prevalencia de "consumo de alcohol de alguna vez a la semana" estimada en este estudio estaría a 1,5 puntos porcentuales de la prevalencia de consumo perjudicial a nivel nacional $(11,07 \%)$ [3]. En el caso de los hombres, estaría a 3 puntos porcentuales de la prevalencia respecto al nivel nacional (17,01\%), pero similar en el de las mujeres y el estimativo nacional (5,47\%). Los jóvenes de 18 a 24 años presentan una prevalencia mayor que el comparador nacional para el mismo grupo de edad $(18,7 \%)$ [3].

Estos datos constituyen una alerta sobre el aumento en los niveles de consumo de alcohol en jóvenes de esta población y sobre la magnitud de este fenómeno.

Vale señalar que en el "Estudio nacional de consumo de sustancias psicoactivas en Colombia 2013" [3] determinan el consumo perjudicial o riesgoso de alcohol, con la población prevalente en el "consumo de alcohol de menos de 30 días", convirtiéndolo en un buen punto de comparación. Es resaltable que, en promedio, los hombres presentaron un consumo 10 puntos porcentuales mayor que las mujeres y 5 puntos porcentuales mayor que el total de la muestra. Esto indicaría que ser hombre por sí mismo implica más prevalencia de consumo de alcohol en cualquier escenario.

Existen pocos estudios con estimaciones de prevalencia de consumo de alcohol en población víctima del conflicto armado [6], y más aún en población escolar y adolescentes. Sin embargo, el trabajo de Hewitt et al. [4] ha encontrado, en población escolar en contexto de posconflicto, presencia de consumo de alcohol moderado del $93 \%$. A su vez, Gantiva et al. [5] estiman la prevalencia de consumo de sustancias psicoactivas en población esco- lar del posconflicto, hallando que el alcohol es la sustancia con mayor porcentaje de prevalencia a lo largo de la vida $(68,8 \%)$ y durante el último mes $(43,4 \%)$.

Por otro lado, un estudio de caracterización epidemiológica en población víctima de desplazamiento forzado en cuatro ciudades, siendo dos del Caribe colombiano, encontró que la prevalencia de consumo de alcohol en el último mes en víctimas adolecentes fue de 7,3\% [7]. De igual manera, el estudio observacional de Andrade et al. [8] halló, en población en situación de desplazamiento forzado, que el $50 \%$ presenta dependencia alcohólica, y el 27,7\%, una dependencia mayor al alcohol.

En cuanto a los factores de riesgo hallados en general, los hombres manifiestan un riesgo tres veces mayor de consumir alcohol que las mujeres, un factor conocido en diferentes estudios $[9,10]$. A su vez, las mujeres de 18 a 24 años presentan un incremento en el riesgo mayor que los hombres del mismo grupo de edad. Esta diferencia se debería a que los hombres inician el consumo de alcohol en edades más tempranas que las mujeres.

Por otra parte, el estudio muestra indicios de que una mala relación con los padres, en especial en el hombre, provoca un riesgo mayor de consumo de alcohol, siendo un factor encontrado en otros estudios [11], lo que denota la relación que hay de los conflictos entre los padres y el adolescente, y el consumo de alcohol [12]. En este último aspecto, el estudio de Cáceres et al. [13], en el que encuestan a 1600 hogares de población desplazada por el conflicto armado en una ciudad del Caribe colombiano, encontró deserción escolar del $20 \%$ de los escolares y $16 \%$ de los adolescentes, escolaridad tardía, pobre desempeño escolar y una proporción importante de niños trabajadores (4 \% de los escolares y $20 \%$ de los adolescentes). A su vez, la pérdida de años escolares, comportamientos agresivos en conducta de matoneo escolar y el consumo de alcohol estarían relacionados [14-16]. En este aspecto, diferentes estudios han establecido los conflictos familiares como un factor psicosocial proclive al consumo de alcohol y otras sustancias psicoactivas, especialmente en edades mayores en los jóvenes [17].

Además, se observó que los jóvenes que consumen algún tipo de sustancia psicoactiva son consumidores de alcohol, siendo consistente con estudios sobre la teoría "puerta de entrada a las drogas" [18], que indican que el uso de alcohol o de tabaco en la adolescencia temprana (12-14 años) sitúa al individuo en gran riesgo de uso posterior e incrementa sus posibilidades de iniciar el consumo de otras drogas de los 18 a los 24 años de edad $[9,19]$. A su vez, los hombres tienen una propensión a consumir alcohol si sus amigos influyen en sus decisiones, como se ha encontrado en otras investigaciones, donde determinan que el consumo por el mejor amigo, seguido por los hermanos, implican respectivamente un riesgo de 13 y 1,8 mayor de consumo de alcohol [20]. 
Por otra parte, los pensamientos suicidas solo fueron un factor de riesgo significativo en las mujeres; en cambio, tener asesorías psicológicas solo es significativo en hombres. En este aspecto, las posibles razones psicológicas apuntan a que, ante un evento negativo, se produce un incremento en los niveles de ansiedad; el individuo reacciona buscando otras alternativas para enfrentar la situación, que en muchos casos se derivan hacia formas poco apropiadas o perjudiciales para la salud, como el consumo de alcohol [21,22]. Este resultado es apoyado por estudios interesados en encontrar las prevalencias de secuelas síquicas en población víctima de violencia y desplazamiento, donde describen la presencia de consumo de alcohol y otras sustancias psicoactivas. En ese sentido, Hewitt et al. [4] encontraron, en población escolar en zona rural del país expuesto al conflicto armado, que el $72 \%$ de la población presentó afectaciones psicológicas. De igual manera, el estudio de Alejo et al. [23] halló que la frecuencia de consumo de alcohol constituye un factor de riesgo entre 1,25 y 4,82 veces de tener trastorno por estrés postraumático en población desplazada por la violencia.

Dentro de las limitaciones del estudio se encuentra las debilidades del instrumento de recolección de la información, debido a que no emplea escalas de consumo de alcohol validadas en el país. Esta debilidad implica no poder describir el consumo nocivo de alcohol en los jóvenes y otros patrones de consumo de alcohol. De igual forma, no es posible conocer antecedentes de consumo de alcohol en el núcleo familiar, siendo un factor importante conocido $[20,24]$. Sin embargo, el tamaño de la muestra de la subregión de los Montes de María permite caracterizar a los jóvenes y sus familias, en una aproximación a las necesidades en salud de población con antecedentes de conflicto armado y persistente incidencia de la pobreza.

Finalmente, este estudio ha mostrado que existen necesidades de atención en salud en población víctima de violencia armada y desplazamiento forzado. El diagnóstico de los factores de riesgo en población joven es un componente esencial y prioritario de la política pública, por la utilidad de esta información en el sector educativo y de la salud, para orientar los programas de promoción y prevención de la salud. Este resultado señala la necesidad de afianzar los programas preventivos sobre consumo de alcohol desde edades más tempranas.

Además, los programas de prevención y promoción que pretendan concienciar a los jóvenes de las consecuencias sociales y en salud por el consumo de alcohol deberían estar articulados con el uso de programas primarios de mejoramiento de la relación familiar y acompañamiento psicosocial.
Todo esto sin perder de vista la posible descomposición del tejido social y las consecuencias psicológicas que pudo dejar el conflicto armado en las relaciones interpersonales en estas poblaciones.

\section{Fuentes de financiación}

Los investigadores del presente estudio participaron en el desarrollo del mismo. Este estudio fue financiado por la Fundación Sersocial. A pesar de lo anterior, los resultados y las conclusiones presentadas en este fueron completamente independientes y ninguno de estos aspectos fue manipulado por el financiador.

\section{Conflicto de interés}

Los autores no presentan conflicto de intereses.

\section{Referencias}

1 World Health Organisation. Global status report on alcohol and health 2014. wHO: Switzerland; 2014.

2 Espada JP, Carrillo FXM, Montesinos MDH. Consumo de alcohol en escolares: descenso de la edad de inicio y cambios en los patrones de ingesta. Adicciones 2000;12(1):57-64.

3 Ministerio del Interior y de Justicia, Ministerio de la Protección Social, Dirección Nacional de Estupefacientes, Observatorio de Drogas de Colombia. Estudio nacional de consumo de sustancias psicoactivas en Colombia 2013. Bogotá: 2014.

4 Hewitt-Ramírez N, Gantiva-Díaz CA, Vera-Maldonado A, Cuervo-Rodríguez MP, Hernández-Olaya NL, Juárez F, et al. Afectaciones psicológicas de niños y adolescentes expuestos al conflicto armado en una zona rural de Colombia. Acta Colomb. Psicol. 2014;17(1):79-89.

5 Gantiva-Díaz CG, Hewitt-Ramírez N, Vera-Maldonado A, Angarita-Varela A, Parado-Baños A, Guillén-Puerto AG. Consumo de sustancias psicoactivas (SPA) en jóvenes de una región en posconflicto armado. Rev. Colomb. Psiquiatr. 2012;41(2):299-308.

6 Castaño GA, Sierra GM, Sánchez D, Semenova N, Salas C, Buitrago C, et al. Salud mental en víctimas de desplazamiento forzado por la violencia en Colombia. El caso de Bogotá, Medellín y Buenaventura. Medellín: Universidad CEs; 2018.

7 Organización Panamericana de la Salud, Instituto Nacional de Salud. Estudio de perfil epidemiológico de población desplazada y población estrato uno no desplazada en cuatro ciudades de Colombia: Montería, Soacha, Cali y Cartagena; 2002.

8 Andrade JA, Bustos JS, Guzmán P. Prevalencia de consumo de alcohol y cigarrillo en personas en situación de desplazamiento forzado en el Tolima. Rev. Psicol. Cient. 2012.

9 Martínez AD, Martínez LRD, Hernández C a, Robles JN, Varela HF, Torres CS. Prevalencia del consumo riesgoso y dañino de alcohol y factores de riesgo en estudiantes universitarios de primer ingreso. Salud Ment. 2008;31(4):271-82.

10 Natera-Rey G, Borges G, Medina-Mora E, Solís-Rojas L, TiburcioSainz M. La influencia de la historia familiar de consumo de alcohol en hombres y mujeres. Salud Pública Mex. 2001;43(1):17-26.

11 Ruiz MR, de Andrade D. La familia y los factores de riesgo relacionados con el consumo de alcohol y tabaco en los niños y ado- 
lescentes (Guayaquil-Ecuador). Rev. Latino-Am. Enfermagem. 2005;13(núm. especial):813-8.

12 Muñoz-Rivas MJ, Graña-López JL. Factores familiares de riesgo y de protección para el consumo de drogas en adolescentes. Psicothema. 2001;13(1):87-94.

13 Cáceres DC, Izquierdo VF, Mantilla L, Jara J, Velandia M. Perfil epidemiológico de la población desplazada por el conflicto armado interno del país en un barrio de Cartagena, Colombia, 2000. Biomedica. 2002;22(Su2):425-44

14 Manrique-Abril FG, Ospina JM, Garcia-Ubaque JC. Consumo de alcohol y tabaco en escolares y adolescentes de Tunja, Colombia, 2009. Rev. Salud Pública. 2011;13(1):89-101.

15 Swahn MH, Donovan JE. Correlates and predictors of violent behavior among adolescent drinkers. J. Adolesc. Health. 2004;34(6):480-92.

16 Swahn MH, Donovan JE. Predictors of fighting attributed to alcohol use among adolescent drinkers. Addict. Behav. 2005;30(7):1317-34

17 Cogollo Z, Arrieta K, Blanco S, Ramos L, Zapata K, Rodríguez Y. Factores psicosociales asociados al consumo de sustancias en estudiantes de una universidad pública. Rev. Salud Pública. 2011;13(3):470-9.
18 Posada-Villa JA, Herazo E, Campo-Arias A. Puerta de entrada al consumo de sustancias ilegales en Colombia: infracciones a la norma de inicio. Rev. Salud Pública. 2009;11(3):406-13.

19 Herrera-Vázquez M, Wagner FA, Velasco-Mondragón E, Borges G, Lazcano-Ponce E. Inicio en el consumo de alcohol y tabaco y transición a otras drogas en estudiantes de Morelos, México. Salud Pública Méx. 2004;46(2):132-40

20 Espada-Sánchez JP, Pereira JR, García-Fernández JM. Influencia de los modelos sociales en el consumo de alcohol de los adolescentes. Psicothema. 2008;20(4):531-7.

21 Aguirre AÁ, Castillo MMA, Zanetti ACG. Consumo de alcohol y autoestima en adolescentes. Rev. Latino-Am. Enfermagem. 2010;18(Spec):634-40

22 Martínez Maldonado R, Pedrão LJ, Alonso Castillo MM, López García KS, Oliva Rodríguez NN. Self-esteem, perceived self-efficacy, consumption of tobacco and alcohol in secondary students from urban and rural areas of Monterrey, Nuevo León, México. Rev. Latino-Am. Enfermagem. 2008;16(Spec):614-20.

23 Alejo EG, Rueda G, Ortega M, Orozco LC. Estudio epidemilógico del trastorno por estrés postraumático en población desplazada por la violencia en Colombia. Univ. Psychol. 2007;6(3):623-35.

24 Landero H, Villarreal G. Consumo de alcohol en estudiantes en relación con el consumo familiar y de los amigos. Psicol. y Salud 2007;17(1):17-23. 